\title{
Correction to: Nature of an Engineered System: Illustrated from Engineering Artefacts and Complex Systems
}

Hillary Sillitto

\section{Correction to: Chapter 36 "Nature of an Engineered System: Illustrated from Engineering Artefacts and Complex Systems " in: G. S. Metcalf et al. (eds.), Handbook of Systems Sciences, https://doi.org/10.1007/978-981-15-0720-5_17}

The grammatical error in the chapter title of Hillary Sillitto's contribution has now been corrected. 